\title{
The role of diagnostic hysteroscopy in diagnosis of incomplete uterine septum/ significant arcuate uterine anomaly in infertile patients in the era of transvaginal 3D ultrasound scan
}

\author{
O. Abuzeid ${ }^{1 *}$, J. LaChance ${ }^{2}$, O. Zaghmout ${ }^{1}$, J. Corrado ${ }^{3}$, J. Hebert ${ }^{1}$, M. Ashraf ${ }^{1,3,4}$ and M. I. Abuzeid ${ }^{1,3,4}$
}

\begin{abstract}
Background: To determine the accuracy of measurement of internal indentation length on transvaginal 3D ultrasound scan (TV 3D US) in detecting patients with an incomplete uterine septum (IUS) or a significant arcuate uterine anomaly (AUA) based on the actual length as measured on diagnostic hysteroscopy. We compared the mean internal indentation length on TV 3D US and on diagnostic hysteroscopy in patients with IUS or a significant AUA.

Results: This is a retrospective study of 546 patients who were found to have IUS (134) or significant AUA (412) on diagnostic hysteroscopy and who had TV 3D US prior to surgery. The mean internal indentation length measured in millimeter on hysteroscopy was significantly higher than the mean internal indentation length measured on TV 3D US in patients with IUS $(16.7 \pm 5.5$ vs $5.5 \pm 4.2 ; P<0.001)$, in patients with significant AUA $(12.9 \pm 2.8$ vs $3.9 \pm$ 4.6; $P<0.001)$, and in the overall population $(13.8 \pm 4.0$ vs $4.3 \pm 4.5 ; P<0.001)$. The same findings were obtaine $\bar{d}$ when the comparison was limited to patients who had moderate significant internal indentation length (10-14 $\mathrm{mm})$ and those with significant internal indentation length (15-25 mm).

Conclusion: Data in this study suggests that the mean internal indentation length in patients with IUS or AUA can be underestimated on TV 3D US. A diagnostic hysteroscopy is the only gold standard to make the correct diagnosis in these patients.
\end{abstract}

Keywords: Arcuate uterine anomaly, Hysteroscopy, Incomplete uterine septum, TV 3D US

\section{Background}

Uterine septum is the most common congenital Müllerian anomaly accounting for more than $50 \%$ of cases [10]. Such anomalies are associated with not only the worst reproductive outcomes but also with excellent results following hysteroscopic surgical correction [13]. In an extensive review of the literature, it was concluded that the septate uterus is the most common uterine anomaly in the infertile population (3.9\%), while the arcuate

\footnotetext{
* Correspondence: omabuzeid@gmail.com

'Department of Obstetrics and Gynecology, Hurley Medical Center and

Michigan State University College of Human Medicine, Two Hurley Plaza, Ste

101, Flint Campus, Flint, MI, USA

Full list of author information is available at the end of the article
}

uterus is the most common anomaly in the general population and in those with recurrent miscarriage [26]. The prevalence of uterine septum and arcuate uterus in infertile patients varies in the literature, but it is estimated to be $3.9 \%$ and $2.1 \%$, respectively [26]. The issue of uterine septum and infertility remains controversial, with conflicting data in the literature [26]. However, the stand of the American Society of Reproductive Medicine (ASRM) is that "There is insufficient evidence to conclude that a uterine septum is associated with infertility" [30]. Furthermore, the association of arcuate uterine anomaly (AUA) and reproductive failure remains controversial with most data suggesting that such anomaly 
is a variant of normal, if the internal indentation length at the fundal midline (IILFM) is less than $10 \mathrm{~mm}$ [30].

ASRM classification of Müllerian anomalies is the most accepted and utilized classification, although it has some limitations, among them the lack of morphometric criteria and its vague description of AUA (class VI) [29]. The latter makes it difficult to distinguish it from subseptate uterus (class $\mathrm{Vb}$ ) [29]. The new guidelines by ASRM define AUA as a uterus with a "depth from the interstitial line to the apex of the indentation $<1 \mathrm{~cm}$ and angle of the indentation $>90^{\circ}$ " [30]. Unfortunately, this new definition does not take into account patients with arcuate appearance and an internal indentation length $\geq$ $1 \mathrm{~cm}$ [30]. If one adopts this definition of AUA, many patients who are found to have an appearance of such anomaly on TV 3D US or hysteroscopy, with an internal indentation length $\geq 10 \mathrm{~mm}$, remain unclassifiable. In addition, the new guidelines by ASRM define subseptate uterus "as having a fundal invagination greater than $1.5 \mathrm{~cm}$ with the central point of the septum at an acute angle $<90^{\circ}$ " [30]. On the other hand, the new classification of Müllerian anomalies proposed by the European Society of Human Reproduction and Embryology and the European Society for Gynecological Endoscopy (ESHREESGE) do not differentiate between AUA and uterine septum. Instead they adopted a definition of uterine septum, if there is an IILFM > 50\% of myometrial wall thickness [12]. Although ESHRE-ESGE classification has been criticized in few reports, it provides the long overdue morphometric criteria needed to make a diagnosis $[16,20$, 21]. The main criticism is over diagnosis that may lead to over treatment. The ESHRE-ESGE was subsequently modified by the Thessaloniki ESHRE/ESGE, which addressed some of the concerns raised by some investigators [11]. A recent publication suggested ESHRE-ESGE cut-off value overestimates the prevalence of septate uterus while that of ASRM underestimates this prevalence, leaving in the gray-zone most of the uteri that experts considered as septate [22]. The authors of this report recommended considering indentation depth $\geq 10 \mathrm{~mm}$ as septate, and this criterion is in agreement with expert opinion [22].

Trans-vaginal 3D ultrasound scan (TV 3D US) has been accepted as a non-invasive, accurate, highly reproducible, and cost-effective diagnostic test for screening at risk patients for congenital uterine anomalies $[8,12$, 14, 30]. This can be achieved by the ability of a TV 3D US to assess both internal and external contour of the uterine fundus, as it enables the operator to visualize the uterus in the coronal plane. Thus, TV 3D US enables us to do away with invasive and more costly procedures such as a combined hysteroscopy and laparoscopy or magnetic resonance imaging (MRI), which have been considered the gold standard, to make the diagnosis of such anomalies. It is now recommended by ASRM that imaging with hysteroscopy should be used to diagnose uterine septa instead of laparoscopy with hysteroscopy, because this approach is less invasive [30]. In addition, both new ASRM guidelines and ESHRE/ESGE classification require TV 3D US $[12,30]$.

Our group has used TV 3D US in screening patients with reproductive failure for uterine septum and arcuate uterine anomaly since 2006 . By 2008 , we started to observe a discrepancy between IILFM as measured by TV 3D US and as measured at time of diagnostic hysteroscopy in patients with IUS and those with AUA [1, 4]. The purpose of this study is to determine the accuracy of measurement of an IILFM on TV 3D US with or without saline infusion hysterogram $(\mathrm{SIH})$ in detecting patients with an IUS or a significant AUA that was diagnosed on diagnostic hysteroscopy based on the actual IILFM.

\section{Methods}

This is a retrospective study of all infertile patients who were found to have IUS or a significant AUA on a diagnostic hysteroscopy performed, as part of their infertility work up, at our unit in the period between January 1, 2008, and October 31, 2017. The study received an exemption from the Institutional Review Board at Hurley Medical Center, Flint, Michigan. The inclusion criterion was to have undergone a TV 3D US with or without $\mathrm{SIH}$ at our unit prior to diagnostic hysteroscopy. Patients who had associated uterine fibroids, endometrial polyps, intrauterine scar tissue, and those with complete septum were excluded. The study included 546 patients who fulfilled such inclusion criteria.

Hysteroscopy was performed at the mid-follicular phase of the menstrual cycle or while the patient was on oral contraceptives. Hysteroscopy was performed under modified general anesthesia. Medications commonly used included Propofol, Versed, and Fentanyl. Hysteroscopy combined with a laparoscopic procedure was performed under general endotracheal anesthesia. All hysteroscopies were performed by the senior author. An ACMI hysteroscope (Division of Olympus; Maple Grove, MN, USA) was used in all patients. Normal saline was used as a distension medium when diagnostic hysteroscopy was performed, while glycine $1.5 \%$ was used if both diagnostic and operative hysteroscopy were performed. We used a pressure infusion bag placed around a 3-1 normal saline or glycine $1.5 \%$ with the pressure of fluid distension ranging between 100 and $120 \mathrm{mmHg}$.

At the time of diagnostic hysteroscopy, the type of uterine anomaly was documented and described according to ASRM classification [29]. The population was classified based on hysteroscopic findings into patients with IUS (134 patients, 24.5\%) [class Vb] and those with significant AUA (412 patients, 75.5\%) [class VI]. For the 
purpose of this study, a diagnosis of an IUS was made, if the central point of indentation was at an acute angle $(<$ $90^{\circ}$ ), did not reach to the region of the internal or external cervical os, and the IILFM measured $\geq 10 \mathrm{~mm}$, while if the central point of indentation was at obtuse angle (> $90^{\circ}$ ) and the IILFM was $\geq 10 \mathrm{~mm}$ the diagnosis of a significant AUA was made [23]. The internal indentation length was measured using a straight resectoscope loop electrode of the ACMI hysteroscope (Fig. 1a) [2]. The black metal tip of the straight resectoscope loop electrode is $5 \mathrm{~mm}$ in length, while the yellow insulating portion of the resectoscope loop is $10 \mathrm{~mm}$ in length (Fig. 1a). The initial measurement was done by advancing a straight loop electrode on one side of the internal indentation up to the tubal ostium (Fig. 1b) [2]. The IILFM was then indirectly calculated to be approximately $60 \%$ of that length (according to Pythagorean Theorem in Mathematics). If an IUS or a significant AUA was divided during the same setting, the actual internal indentation length was measured directly (Fig. 1c) [2]. Based on internal indentation length on hysteroscopy, the patients with IUS or significant AUA anomalies were further divided into 2 main groups, those with moderate internal indentation length (10-14 $\mathrm{mm})$ and those with significant internal indentation length ( $\geq 15 \mathrm{~mm}$ ).

Before hysteroscopy an initial transvaginal 2D ultrasound scan (TV 2D US) assessment of the pelvis was performed, as part of infertility work up, to exclude any adnexal pathology such as endometrioma, hydrosalpinx, or any uterine pathology, such as uterine fibroid and endometrial polyps. In addition, TV 2D US was used to determine the orientation of the uterus and to facilitate insertion of a catheter into the cervical canal for instillation of the saline at time of SIH. The study was performed at the mid-follicular phase of the menstrual cycle or while the patient was on oral contraceptives. This was followed by a TV 3D US with or without SIH utilizing a Medison Sonace 8000 US machine (Medison, Seoul 135-280, Korea) and an endocavitary probe RIC59H 5-9 MHz. The uterus was then visualized by TV 3D
US in a strict mid-sagittal view, adjusting the capture window to obtain the optimal 3D US volume. The 3D US volume was then obtained, using the automatic sweep of the mechanical transducer, at angle of $90^{\circ}$ from one side of the uterus to the other, bisecting the capture plan. If there was any doubt about the quality or completeness of the TV 3D US volume, the acquisition was repeated. The dataset was then stored to the hard drive of the ultrasound machine.

The dataset was adjusted to ensure that the endometrium was evident in the three planes and that the fundal aspect of the uterus and the endometrial cavity were demonstrable in the coronal plane, and the interstitial portions of both fallopian tubes were displayed simultaneously. This enabled us to assess the uterine morphology and shape of the uterine cavity in the coronal plane. Saline infusion hysterogram ( $\mathrm{SIH})$ was performed as part of the evaluation of infertility using sterile normal saline using either a Rocket intrauterine insemination catheter or a hysterosalpingogram (HSG) catheter as described before [5]. Preoperatively, 104 patients (19.0\%) had only TV 3D US, 62 patients (11.4\%) had only TV 3D US with $\mathrm{SIH}$, and 380 patients $(69.6 \%)$ had both studies. Any internal indentation in the endometrial cavity was calculated on TV 3D US with or without SIH. This involved measuring the distance from the midpoint of the line joining the internal tubal ostia and the bottom of indentation of the cavity (Fig. 2a and Fig. 3a with their corresponding hysteroscopic appearance, Fig. 2b and Fig. 3b). This represented the IILFM. If the two values were available on TV 3D US, and TV 3D US with SIH, a mean value was obtained; otherwise, the one available value was used. Based on internal indentation length on TV 3D US, the patients were divided into 4 subgroups. The internal indentation length of subgroups $1,2,3$, and 4 were $0.00 \mathrm{~mm}, 1-4.9 \mathrm{~mm}, 5-9.9 \mathrm{~mm}$, and $\geq 10 \mathrm{~mm}$, respectively.

We compared internal indentation length on hysteroscopy to the internal indentation length on TV 3D US with or without SIH in patients with IUS, significant

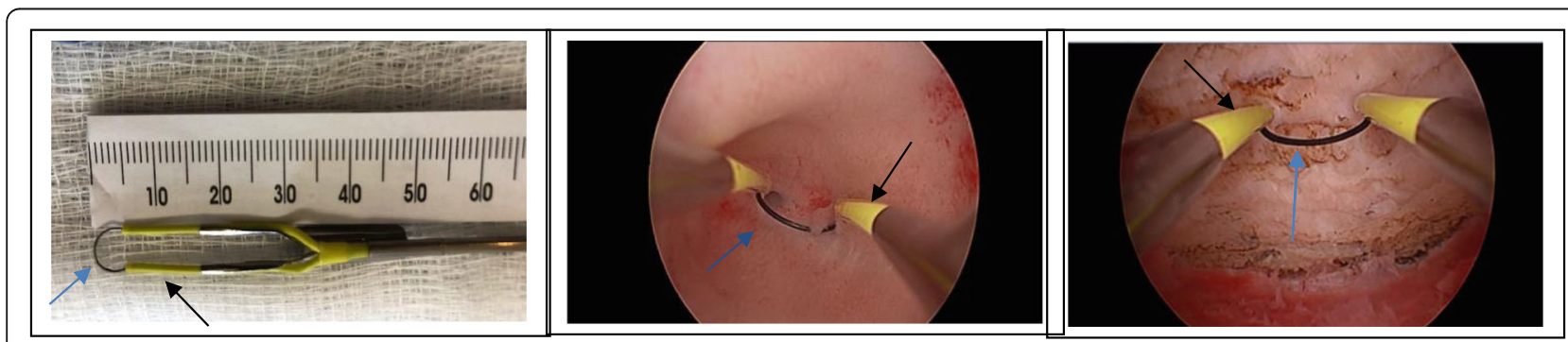

Fig. 1 a A picture that illustrates the length of various parts of a straight resectoscope loop electrode of the ACMI hysteroscope. $\mathbf{b}$ A picture that illustrates how a straight resectoscope loop electrode was used to indirectly measure the internal indentation length. c A picture that illustrates how a straight resectoscope loop electrode was used to directly measure the internal indentation length. $\mathbf{a}-\mathbf{c}$ The blue arrow is pointed to the metal electrode, $5 \mathrm{~mm}$ in length, and the black arrow is pointed to the yellow insulator, $10 \mathrm{~mm}$ in length after the septum was divided 


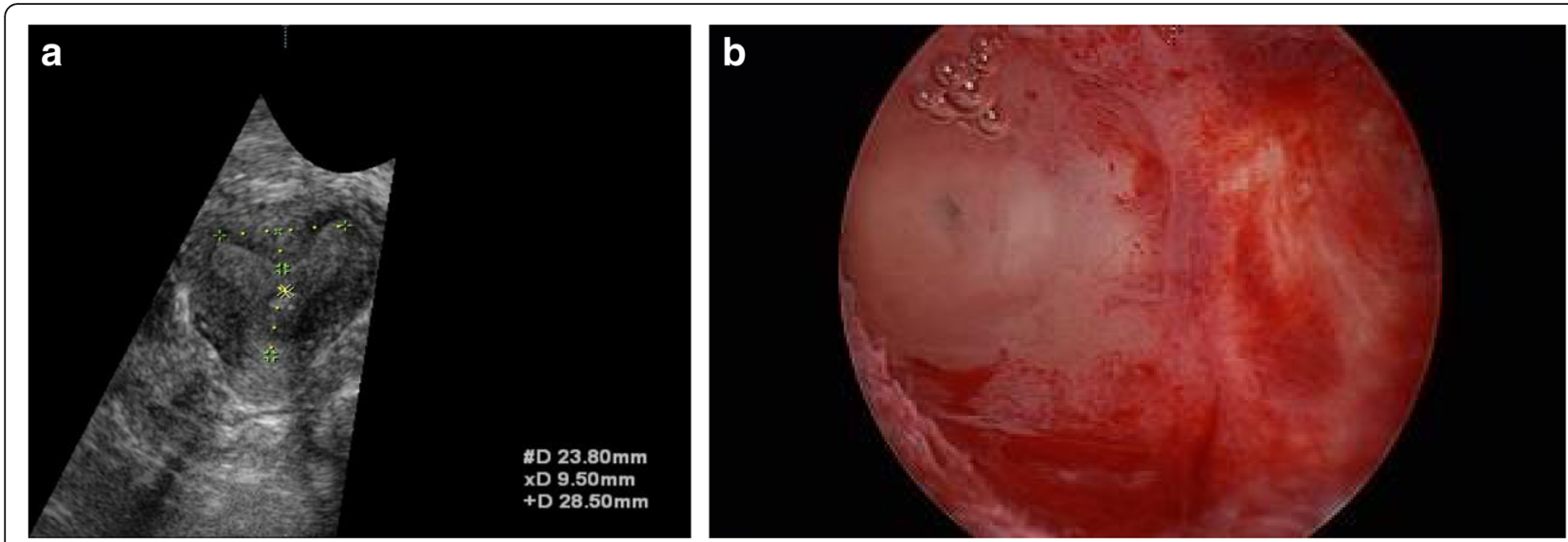

Fig. 2 a A picture that illustrates how the internal indentation length (IILFM) was measured on TV 3D US by measuring the distance from the midpoint of the line joining the internal tubal ostia and the bottom of indentation of the cavity in a patient with an incomplete uterine septum. b A picture that illustrates the corresponding hysteroscopic appearance of the uterine fundus of the patient whose TV 3D US is illustrated in Fig. 2a

AUA and the overall population. In addition, based on the findings on hysteroscopy, this comparison was repeated for patients with moderate internal indentation length $(10-14 \mathrm{~mm})$ and those with significant internal indentation length $(\geq 15 \mathrm{~mm})$. We also calculated the incidence of all subgroups (1-4) in patients with IUS and AUA in the total population, in patients with moderate significant, and in those with significant internal indentation length. Statistical analysis was performed using Student's paired $T$ test, chi-square analysis, and correlation analysis where appropriate.

\section{Results}

Table 1 summarizes demographic data. The mean IILFM measured in millimeter on hysteroscopy was significantly higher than the IILFM measured on TV 3D US with or without $\mathrm{SIH}$, in patients with IUS $(16.7 \pm 5.5$ vs $5.5 \pm$ 4.2; $P<0.001)$, in patients with significant AUA (12.9 \pm 2.8 vs $3.9 \pm 4.6 ; P<0.001)$, and in the overall population $(13.8 \pm 4.0$ vs $4.3 \pm 4.5 ; P<0.001)$ (Fig. 4). A correlation analysis between the findings on hysteroscopy and the findings on TV 3D US revealed a moderate correlation for IUS $(r=0.436, P<0.001)$ and a weak correlation for patients with significant AUA $(r=0.192, P<0.001)$ and a weak correlation for the overall population $(r=0.299$, $P<0.001$ ).

The data was then analyzed for patients who only had a moderate internal indentation length on hysteroscopy (length $10-14 \mathrm{~mm}$ ). The mean IILFM measured in millimeter on hysteroscopy was significantly higher than that measured on TV 3D US with or without SIH, in patients with IUS $(11.2+1.2$ vs $3.7+3.0 ; P<0.001)$, in patients
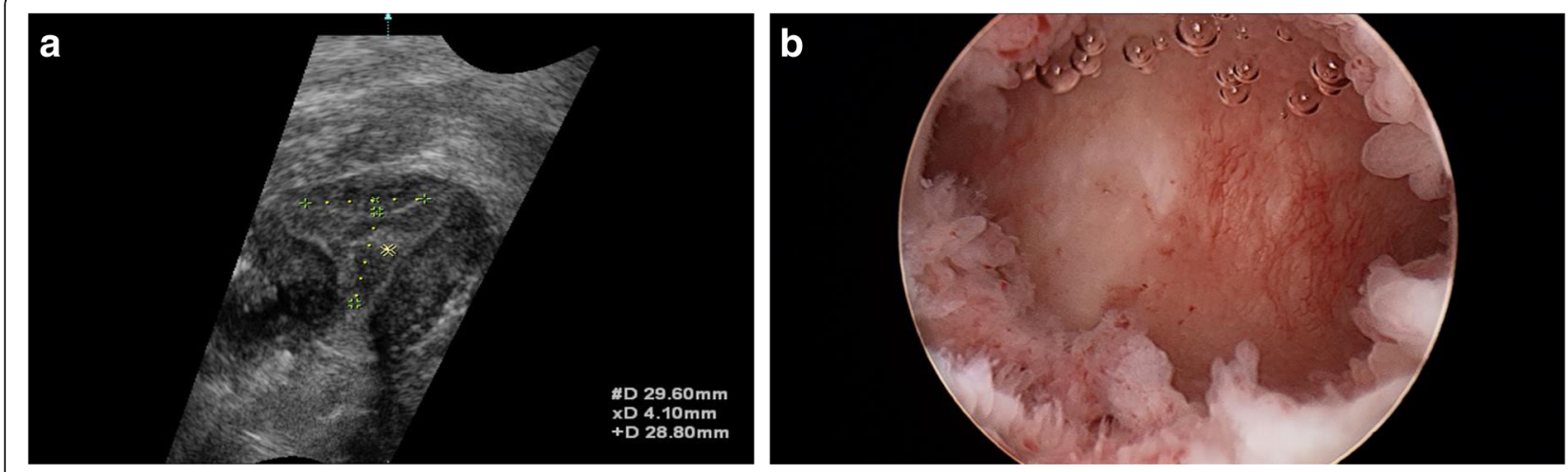

Fig. 3 a A picture that illustrates how the internal indentation length (IILFM) was measured on TV 3D US by measuring the distance from the midpoint of the line joining the internal tubal ostia and the bottom of indentation of the cavity in a patient with a significant arcuate uterine anomaly. $\mathbf{b}$ A picture that illustrates the corresponding hysteroscopic appearance of the uterine fundus of the patient whose TV 3D US is illustrated in Figure 3a 
Table 1 Demographic data

\begin{tabular}{|c|c|c|c|c|}
\hline & $\begin{array}{l}\text { Incomplete uterine septum } \\
\text { No. }=134\end{array}$ & $\begin{array}{l}\text { Significant arcuate uterine anomaly } \\
\text { No. }=412\end{array}$ & $P$ value & $\begin{array}{l}\text { Overall total population } \\
\text { No. }=546\end{array}$ \\
\hline Age (years) & $31.6 \pm 4.6$ & $32.5 \pm 5.0$ & 0.052 & $32.5 \pm 5.0$ \\
\hline Duration of infertility (years) & $2.8 \pm 2.1$ & $3.0 \pm 2.3$ & NS & $2.9 \pm 3.0$ \\
\hline$\%$ Primary infertility & $73.4 \%$ & $71.0 \%$ & NS & $71.6 \%$ \\
\hline $\mathrm{BMI}\left(\mathrm{kg} / \mathrm{m}^{2}\right)$ & $26.4 \pm 9.2$ & $26.2 \pm 8.0$ & NS & $26.3 \pm 8.5$ \\
\hline Day 3 FSH (mlU/ml) & $6.6 \pm 3.3$ & $7.0 \pm 3.4$ & NS & $7.0 \pm 3.5$ \\
\hline Day $3 \mathrm{LH}(\mathrm{mlU} / \mathrm{ml})$ & $5.8 \pm 3.4$ & $6.1 \pm 3.8$ & NS & $6.0 \pm 3.7$ \\
\hline Prolactin (ng/ml) & $12.7 \pm 8.1$ & $14.5 \pm 13.2$ & NS & $14.3 \pm 12.6$ \\
\hline TSH (ulU/ml) & $2.0 \pm 1.7$ & $2.0 \pm 1.6$ & NS & $2.0 \pm 1.6$ \\
\hline$\%$ With history of miscarriage & $15.5 \%$ & $19.8 \%$ & NS & $18.7 \%$ \\
\hline$\%$ Male infertility & $34.2 \%$ & $38.9 \%$ & NS & $37.0 \%$ \\
\hline$\%$ Ovulatory disorder & $32.3 \%$ & $29.2 \%$ & NS & $28.4 \%$ \\
\hline \%Tubal factors & $23.7 \%$ & $27.7 \%$ & NS & $26.2 \%$ \\
\hline$\%$ Endometriosis & $52.6 \%$ & $46.7 \%$ & NS & $48.8 \%$ \\
\hline
\end{tabular}

with significant AUA $(11.0+1.1$ vs $3.2+2.8 ; P<0.001)$, and in the overall population $(11.0 \pm 1.2$ vs $3.2 \pm 2.8 ; P$ $<0.001$ ) (Fig. 5). A correlation analysis between the findings on hysteroscopy and the findings on TV 3D US revealed no statistically significant correlation for IUS $(r=$ $0.175, P=0.315)$ and weak statistically correlations for AUA $(r=0.143, P<0.0)$ and for the overall population $(r=0.151, P<0.05)$.

The data was then analyzed for patients who only had a significant internal indentation length on hysteroscopy $(\geq 15 \mathrm{~mm})$. The mean IILFM measured in millimeter on hysteroscopy was significantly higher than that measured on TV 3D US with or without SIH, in patients with IUS $(18.6 \pm 5.2$ vs $6.1 \pm 4.4 ; P<0.001)$, in patients with significant AUA $(15.7 \pm 2.1$ vs $5.0 \pm 6.3 ; P<0.001)$, and in the overall population $(16.8 \pm 3.8$ vs $5.4 \pm 5.6 ; P<0.001)$ (Fig. 6). A correlation analysis between the findings on hysteroscopy and the findings on TV 3D US revealed a low-moderate correlation for IUS $(r=0.396, P<0.001)$, no statistically significant correlation for significant AUA $(r=0.034, P=0.668)$, and a weak correlation for the overall population $(r=0.202, P<0.01)$.

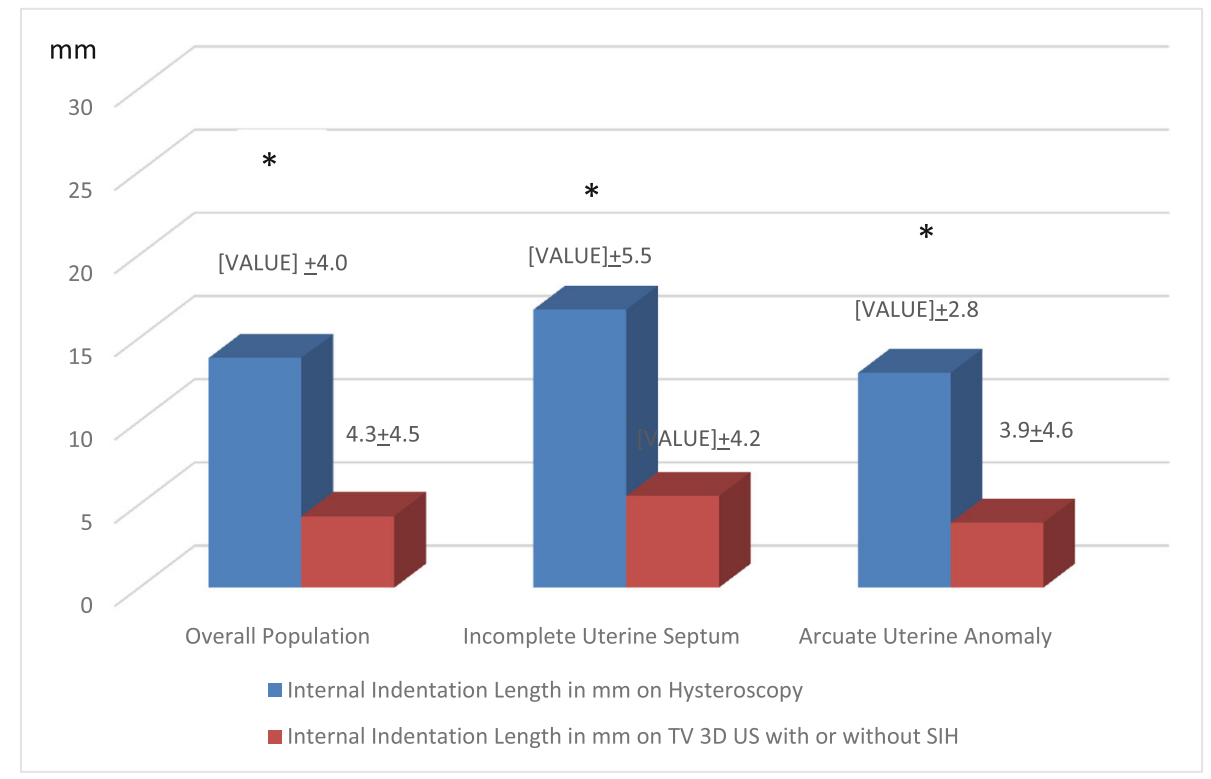

$* \mathrm{P}<0.001$

Fig. 4 Comparison between the mean IILFM measured in millimeter on hysteroscopy and on TV 3D US with or without SIH, in patients with IUS, significant AUA, and in the overall population in all patients 


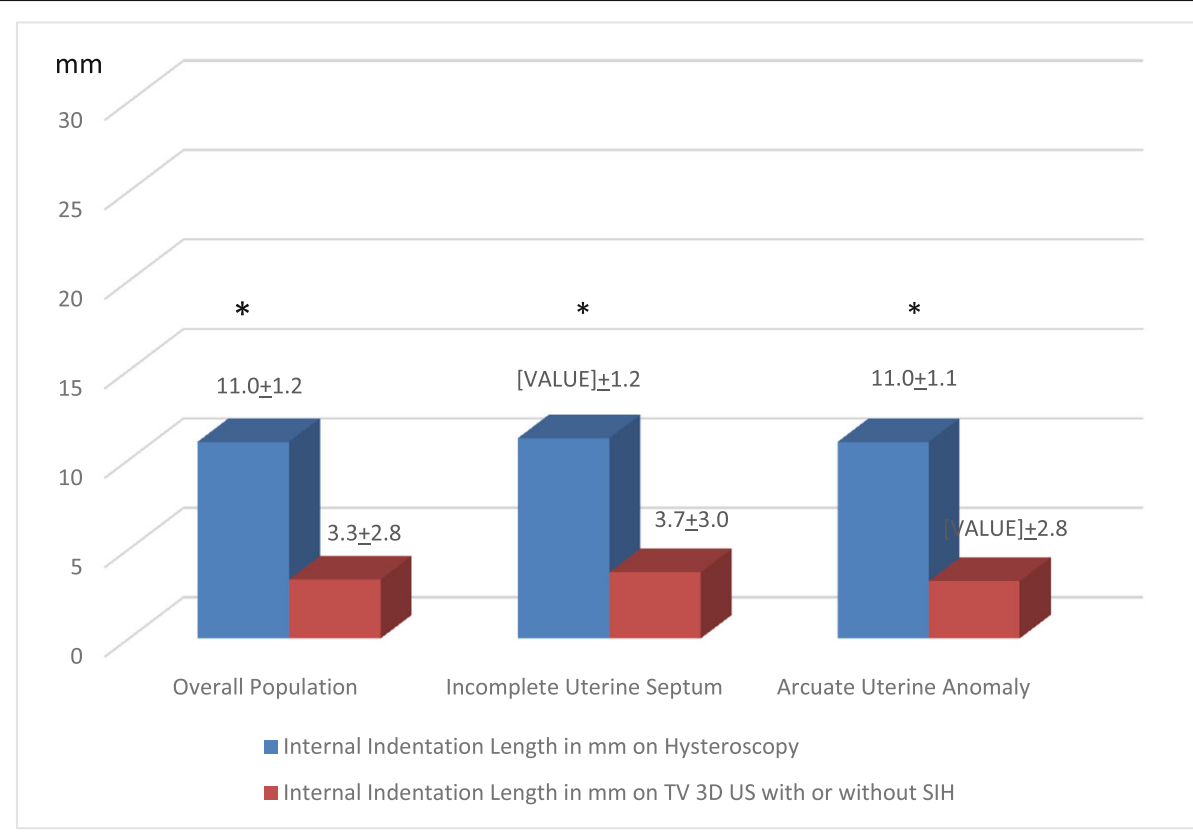

$* \mathrm{P}<0.001$

Fig. 5 Comparison between the mean IILFM measured in mm on hysteroscopy and on TV 3D US with or without SIH, in patients with IUS, significant $A \cup A$, and in the overall population in patients with moderate internal indentation length $(10-14 \mathrm{~mm})$

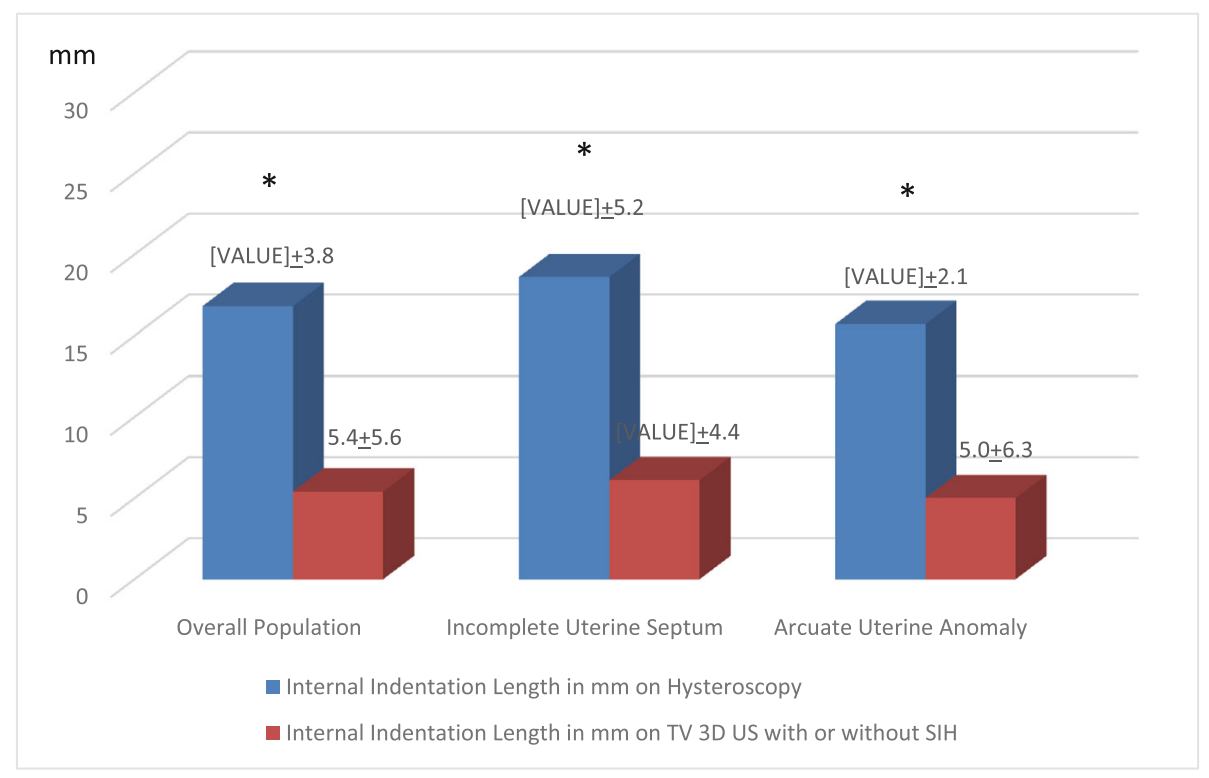

$* \mathrm{P}<0.001$

Fig. 6 Comparison between the mean IILFM measured in mm on hysteroscopy and on TV 3D US with or without SIH, in patients with IUS, significant AUA, and in the overall population in patients with significant internal indentation length ( $\geq 15 \mathrm{~mm}$ ) 
Fifty-two percent of patients had moderate internal indentation length on hysteroscopy, while $48.0 \%$ had significant internal indentation length. There was no evidence of any internal indentation length $(0.00 \mathrm{~mm})$ on TV 3D US with or without SIH in 161 patients (29.5\%) [subgroup 1]. The internal indentation length on TV 3D US with or without SIH was found to be 1-4.9 $\mathrm{mm}$ in 159 patients (29.1\%) [subgroup 2]. The internal indentation length on TV 3D US with or without SIH was found to be $5-9.9 \mathrm{~mm}$ in 199 patients (36.4\%) [subgroup 3]. The internal indentation length on TV 3D US with or without SIH was found to be $\geq 10 \mathrm{~mm} 27 \mathrm{pa}$ tients (4.9\%) [subgroup 4].

Table 2 illustrates that in patients with a moderate internal indentation $(10-14 \mathrm{~mm})$ on hysteroscopy, there was no significant difference in percentage of patient who had IUS vs AUA irrespective of the length of the internal indentation on TV 3D US in 4 subgroups. The majority of such patients $(71.8 \%)$ had either no internal indentation $(36.6 \%)$ or very subtle internal indentation $(1-4.9 \mathrm{~mm})$ [35.2\%] on TV 3D US. The remaining patients $(28.2 \%)$ had an internal indentation on TV 3D US that was between 5 and $9.9 \mathrm{~mm}(27.1 \%)$ or $\geq 10 \mathrm{~mm}$ (1.1\%). Table 3 illustrates that in patients with a significant internal indentation ( $\geq 15 \mathrm{~mm}$ ) on hysteroscopy, there was no significant difference in percentage of patients who had IUS vs AUA irrespective of the length of the internal indentation on TV 3D US in the 4 subgroups. The majority of such patients $(55.7 \%)$ had either an internal indentation on TV 3D US that was between 5 and $9.9 \mathrm{~mm} \mathrm{(46.5 \% )}$ or $\geq 10 \mathrm{~mm} \mathrm{(9.2 \% ).} \mathrm{The}$ remaining patients $(44.3 \%)$ had no internal indentation on TV 3D US (21.8\%) or very subtle internal indentation $(1-4.9 \mathrm{~mm})$ [22.5\%].

\section{Discussion}

This is the first study to suggest that TV 3D US is not accurate in measuring the internal indentation length in patients with IUS or AUA when compared to a diagnostic hysteroscopy. Measurement on TV 3D US consistently underestimates the IILFM compared to the actual measurement on diagnostic hysteroscopy. Such findings were confirmed irrespective of the type of the anomaly or the actual length of the internal indentation as measured on diagnostic hysteroscopy. Patients with moderate internal indentation length $(10-14 \mathrm{~mm})$ and those with significant internal indentation length $(\geq 15 \mathrm{~mm})$ on diagnostic hysteroscopy had such lesions underestimated on TV 3D US with or without SIH. In turn, our data suggests that there may be an element of uterine factor in some infertile patients that goes undiagnosed if one relies on TV 3D US with or without SIH. Such uterine anomalies may also be found in some patients with unexplained infertility. It may also explain a possible etiology for repeated implantation failure after various infertility treatments including IVF-ET. The presence of uterine septum has been linked to repeated implantation failure [25]. In addition, our findings may in part explain the variable incidence of uterine septum and AUA in infertile patients in the literature [26]. It is not clear as to why the measurement of the internal indentation length of IUS and AUA on TV 3D US is not accurate. However, volume transvaginal ultrasound pictures are computer generated, and therefore, it is possible that the IILFM in the generated pictures is not accurate.

Published data in the literature suggests that TV 3D US is the gold standard for the diagnosis of septate, subseptate, and AUA with high degree of sensitivity and specificity [8, 14]. However, most of this literature does not clearly differentiate between patients with complete septum and IUS, and between those with subtle and significant IUS [9]. Patients with complete uterine septum and those with significant IUS could arguably be suspected on TV 2D US (in transverse view) or even on HSG, albeit such tests cannot differentiate between such anomalies, and complete or incomplete bicornuate uterus because of lack of evaluation of the external fundal contour [31]. In addition, the data in the literature on the use of TV 3D US for the diagnosis of AUA is both limited and confusing. Such studies may have also included patients with subtle arcuate uterine anomaly of no clinical significance. In a study by Graupera et. al. 2015, that compared TV 3D US to MRI in diagnosis of Müllerian anomalies, using the ESHRE-ESGE consensus, all patients had a significant pathology as they were initially suspected on TV 2D US [9] Moini et. al. 2013.

Table 2 Percentage of patients with IUS and AUA and moderate internal indentation (10-14 mm) on hysteroscopy, in 4 subgroups based on the internal indentation length on TV 3D US with or without SIH

\begin{tabular}{|c|c|c|c|c|}
\hline & $\begin{array}{l}\text { Incomplete uterine septum } \\
\text { No. }=35\end{array}$ & $\begin{array}{l}\text { Significant arcuate uterine anomaly } \\
\text { No. }=249\end{array}$ & $P$ value & $\begin{array}{l}\text { Overall total population } \\
\text { No. }=284\end{array}$ \\
\hline Subgroup 1, IILFM (0.00 mm) on TV 3D US & $11(31.4 \%)$ & $93(37.3 \%)$ & NS & $104(36.6 \%)$ \\
\hline Subgroup 2, IILFM (-4.9 mm) on TV 3D US & $13(37.1 \%)$ & $87(34.9 \%)$ & NS & $100(35.2 \%)$ \\
\hline Subgroup 3, IILFM (5-9.9 mm) on TV 3D US & $9(25.7 \%$ & $68(27.3 \%)$ & NS & $77(27.1 \%)$ \\
\hline Subgroup 4, IILFM ( $\geq 10 \mathrm{~mm})$ on TV 3D US & $2(5.7 \%)$ & $1(0.4 \%)$ & NS & $3(1.1 \%)$ \\
\hline
\end{tabular}


Table 3 Percentage of patients with IUS and AUA and a significant internal indentation (> $15 \mathrm{~mm}$ ) on hysteroscopy, in 4 subgroups based on the internal indentation length on TV 3D US with or without SIH

\begin{tabular}{|c|c|c|c|c|}
\hline & $\begin{array}{l}\text { Incomplete uterine septum } \\
\text { No. }=99\end{array}$ & $\begin{array}{l}\text { Significant Arcuate uterine anomaly } \\
\text { No. }=163\end{array}$ & $P$ value & $\begin{array}{l}\text { Overall total population } \\
\text { No. }=262\end{array}$ \\
\hline Subgroup 1, IILFM (0.00 mm) on TV 3D US & $18(18.2 \%)$ & $39(23.9 \%)$ & NS & $57(21.8 \%)$ \\
\hline Subgroup 2, IILFM (1-4.9 mm) on TV 3D US & $17(17.2 \%)$ & $42(25.8 \%)$ & NS & $59(22.5 \%)$ \\
\hline Subgroup 3, IILFM (5-9.9 mm) on TV 3D US & $51(51.5 \%)$ & $71(43.6 \%)$ & NS & $122(46.5 \%)$ \\
\hline Subgroup 4, IILFM ( $\geq 10 \mathrm{~mm}$ ) on TV 3D US & $13(13.1 \%)$ & $11(6.7 \%)$ & NS & $24(9.2 \%)$ \\
\hline
\end{tabular}

compared the findings on TV 3D US to hysteroscopy and laparoscopy in patients with suspected uterine septum and reported that TV 3D US was more accurate in long complete septum and less accurate in IUS and AUA [24]. The later findings are similar to that in our study.

Several publications, albeit with small sample size, that reported on the accuracy of TV 3D US in diagnosis of IUS or AUA relied on comparison to office hysteroscopy $[7,8,17]$. Many investigators found office hysteroscopy to be a valuable method to screen for congenital anomalies of the uterine cavity [7, 8, 17]. However, we believe that such subtle anomalies can be missed if uterine distension is not adequate, as commonly happens during office hysteroscopy to avoid patients' discomfort [19, 27, 28]. A recent video abstract suggested that even a diagnostic hysteroscopy, performed under modified general anesthesia, can miss the diagnosis of such anomalies if uterine distension is suboptimal [3]. In addition, the narrow and small view on office hysteroscopy can make the evaluation for such anomalies difficult, especially if the patient is uncomfortable. Furthermore, a study that reported on the reproducibility of diagnosing intrauterine abnormalities through office hysteroscopy, found that the interobserver agreement appeared to be disappointing [15]. In a study by Smit et. al. 2013, it was shown that in infertile patients the international agreement on the diagnosis of the septate uterus and arcuate uterus by office hysteroscopy appeared to be rather disappointing [27]. The same findings were found in a subsequent study by the same authors even when some diagnostic criteria were used at time of office hysteroscopy [28]. Therefore, the consensus on the accuracy of office hysteroscopy during assessment of the uterine shape seemed to be poor, especially for the less profound variations. However, the suggestion that office hysteroscopy is less accurate than a diagnostic hysteroscopy under sedation in diagnosis of IUS and AUA can only be confirmed by a prospective comparative study.

In a prospective study of patients with recurrent pregnancy loss, who were suspected to have septate, subseptate, and AUA, TV 3D US was extremely accurate in making the diagnosis of such anomalies, as confirmed on subsequent diagnostic hysteroscopy and laparoscopy
[8]. In the same study, the authors reported that a negative study on TV 3D US was also accurate in ruling out such anomalies as confirmed on subsequent office hysteroscopy [8]. In contrast, $29.5 \%$ of the patients in our study were found to have no evidence of any internal indentation on TV 3D US with or without SIH $(0.00 \mathrm{~mm})$ [subgroup 1]. Therefore, our findings are not in agreement with those of Ghi et. al. 2009 [8]. If the recommendation by Ghi et. al. 2009 [8] is followed, $29.5 \%$ of the patients in our study (subgroup 1) would have been considered to have no IUS or significant AUA based on TV 3D US. In addition, another $29.1 \%$ of the patients in our study with IILFM of 1-4.9 mm (subgroup 2) would have also been considered normal with respect to such Müllerian anomaly based on TV 3D US. Furthermore, an additional $36.4 \%$ of the patients in our study with IILFM of 5-9.9 mm (subgroup 3) would also have been considered a variant of normal based on TV 3D US. This group of patients (subgroup 3) with IILFM of 5-9.9 mm on TV 3D US would have been considered to have insignificant internal indentation length, irrespective of its appearance, according to recent ASRM guidelines [30]. In such patients, a hysteroscopy would not be recommended and in turn the diagnosis would have been missed. On the other hand, these patients (subgroup 3) would be considered to have an IUS, irrespective of its appearance, according to ESHRE/ESGE classification [12]. All in all, in only $4.9 \%$ of our patients, TV 3D US with or without SIH would have revealed an internal indentation length of $\geq 10 \mathrm{~mm}$ (subgroup 4) and in turn a correct diagnosis would have been made according to the criteria used in our study providing that one does not follow the recent ASRM guidelines, which suggest that IUS is defined as an internal indentation length of $\geq$ $15 \mathrm{~mm}$. While in $95.4 \%$ of the population studied, TV 3D US would have been most likely interpreted as normal or a variant of normal for such Müllerian anomaly [30]. It is worth of note that even if the new classification of ESHRE/ESGE regarding uterine septum (IILFM more $>50 \%$ of myometrial thickness) was used in our study, only $41.0 \%$ of the patients (subgroup 3 and subgroup 4) would have been suspected [12].

It is important to discuss our findings in context with the recent guidelines of ASRM and ESHRE/ESGE 
classification [12, 30]. Some of our patients with AUA and internal indentation length of 5-9.9 mm (subgroup 3) on TV 3D US would have been diagnosed as arcuate uterus, but it would have been thought to be of no clinical significance, based on the recent ASRM guidelines [30]. On the other hand, all the patients in our study who had AUA with IILFM $\geq 10 \mathrm{~mm}$ on hysteroscopy would have been considered unclassifiable by the recent guidelines of ASRM 2016. In that context, it is worth of note that some investigators defined AUA as those with arcuate appearance and indentation length to be between 1.0 and $1.5 \mathrm{~cm} \mathrm{[18].} \mathrm{In} \mathrm{contrast,} \mathrm{all} \mathrm{the} \mathrm{patients} \mathrm{in}$ our study would have been considered to have an IUS based on ESHRE/ESGE classification [12].

It should be emphasized that TV 3D US is an essential part of work-up of infertile patients. The value of TV 3D US in the diagnosis of IUS and AUA is well established, and therefore, it is a mandatory step in the assessment of the uterine cavity in patients with a suspected IUS, AUA, or bicornuate uterus, especially before planning operative hysteroscopy. TV 3D US is a noninvasive and a reproducible method that can provide information about both the external contour and the uterine cavity at the same time. Hysteroscopy alone (without laparoscopy) cannot differentiate between septate and bicornuate uterus and should only be performed after TV 3D US is done in such patients.

Our study has its limitations. First, it is a retrospective study with all the limitation related to this design. Second is the fact that TV 3D UD was performed in the follicular phase. Many authors suggested better view in the luteal phase as the endometrium is more prominent [8]. However, in our experience we did not find that to be a limiting factor to have good visualization of the endometrial cavity. Another limitation in our study is the fact that the technique used to measure the internal indentation length at the fundal midline (IILFM) has not been validated and it may not be perfectly accurate. However, even if that is the case, the difference may be a few millimeter and in turn may not impact the overall conclusions of this study. More studies are needed to confirm our finding. In future studies, the use of a novel graduated intrauterine palpator described recently by Di Spiezio et. al. 2016 [6] can enhance the accuracy of measurement of IILFM on hysteroscopy. Another limitation is the operator bias, as all surgeries were performed by the senior author, who was aware of the TV 3D US findings prior to surgery. On the other hand, this may be considered a strength of the study. Despite the negative results on TV 3D US, the operator was not influenced by such findings at the time of hysteroscopy. The strength of this study stems from the fact that all the data is from one center, which eliminates variability in diagnostic measures by the operators. In addition, another area of strength is the large sample size included in this study.

\section{Conclusion}

Data in this study suggests that mean IILFM in patients with IUS or AUA can be underestimated on TV 3D US. It appears that a diagnostic hysteroscopy by an experienced reproductive surgeon is the only gold standard to make the correct diagnosis in these patients. Our findings may have tremendous implications in management of infertility patients. Additional prospective studies are needed to confirm our findings.

\section{Abbreviations \\ AUA: Arcuate uterine anomaly; HSG: Hysterosalpingogram; IILFM: Internal indentation length at the fundal midline; IUS: Incomplete uterine septum; MRI: Magnetic resonance imaging; SIH: Saline infusion hysterogram; TV 2D US: Transvaginal 2D ultrasound scan; TV 3D US: Transvaginal 3D ultrasound scan}

\section{Acknowledgements}

Not applicable

\section{Authors' contributions}

OA contributed to the protocol development, data collection, data analysis, and manuscript writing. JLC contributed to the protocol development, data analysis, manuscript editing, and final approval of manuscript. OZ contributed to the data collection, manuscript editing, and final approval of manuscript. JC contributed to the provision of study patients, performing ultrasound, manuscript editing, and final approval of contributed to the manuscript. JH contributed to the manuscript editing and final approval of manuscript. MA contributed to the provision of study patients, manuscript editing, final approval of manuscript. MIA contributed to the protocol development, provision of study patients, data collection, data analysis, manuscript editing, and final approval. All authors read and approved the final manuscript.

Funding

The study did not receive external funding.

Availability of data and materials

Not applicable

Ethics approval and consent to participate

The study received an exemption from the Institutional Review Board at Hurley Medical Center, Flint, Michigan.

Consent for publication

Not applicable

\section{Competing interests}

The authors state explicitly that there are no conflicts of interest in connection with this article. All authors (OA, JLC, OZ, JC, JH, MA, MIA) declare independence from any funding agency for this work.

\section{Author details}

${ }^{1}$ Department of Obstetrics and Gynecology, Hurley Medical Center and Michigan State University College of Human Medicine, Two Hurley Plaza, Ste 101, Flint Campus, Flint, MI, USA. ²Department of Research, Hurley Medical Center and Michigan State University College of Human Medicine, Flint Campus, Flint, MI, USA. 'IVF Michigan Rochester Hills and Flint PC, Rochester Hills, MI, USA. ${ }^{4}$ Division of Reproductive Endocrinology and Infertility, Department of Obstetrics and Gynecology, Hurley Medical Center and Michigan State University College of Human Medicine, Flint Campus, Flint, MI, USA. 
Received: 9 August 2019 Accepted: 11 November 2019

Published online: 14 January 2020

\section{References}

1. Abuzeid M, Abuzeid O (2014). Three-dimensional ultrasonography of subtle uterine anomalies: correlation with hysterosalpingogram, two-dimensional ultrasonography, and hysteroscopy. Ultrasound in Gynecology. Editors: Botros Rizk and Elizabeth Puscheck. Cambridge University Press, Chapter 8, 66-79.

2. Abuzeid M, Ghourab G, Abuzeid O et al (2014) Reproductive outcome after IVF following hysteroscopic division of incomplete uterine septum/arcuate uterine anomaly in women with primary infertility. Facts Views Vis Obgyn 6(4):194-202

3. Abuzeid O, Raju R, Hebert J et al (2016) Effect of proper uterine distention on the detection rate of subtle uterine anomalies during hysteroscopy. JMIG 23(7):S134-S135

4. Abuzeid O, Zaghmout O, Corrado J et al (2015) Comparison between the findings on trans-vaginal 3D ultrasound scan and hysteroscopy in patients diagnosed with subtle incomplete uterine septum/arcuate uterine anomaly on hysteroscopy. Fertil Steril 104(3):e28 0-70

5. Bolnick A, Abuzeid M (2017). Saline infusion sonohysterography (SIS) In: Standard Operational Procedures in Reproductive Medicine Laboratory and Clinical Practice. Editor: Botros Rizk. CRC Francis \& Taylor Group, Section 2, Chapter 38, 98-99.

6. Di Spiezio SA, Zizolfi B, Bettocchi S et al (2016) Accuracy of hysteroscopic metroplasty with the combination of presurgical 3-dimensional ultrasonography and a novel graduated intrauterine palpator: a randomized controlled trial. JMIG 23(4):557-566

7. Faivre E, Fernandez H, Deffieux X et al (2012) Accuracy of three-dimensional ultrasonography in differential diagnosis of septate and bicornuate uterus compared with office hysteroscopy and pelvic magnetic resonance imaging. JMIG 19(1):101-106

8. Ghi T, Casadio P, Kuleva M et al (2009) Accuracy of three-dimensional ultrasound in diagnosis and classification of congenital uterine anomalies. Fertil Steril 92(2):808-813

9. Graupera B, Pascual MA, Hereter L et al (2015) Accuracy of threedimensional ultrasound compared with magnetic resonance imaging in diagnosis of Mullerian duct anomalies using ESHRE-ESGE consensus on the classification of congenital anomalies of the female genital tract. Ultrasound Obstet Gynecol 46:616-622

10. Grimbizis G, Camus M, Tarlatzis BC et al (2001) Clinical implications of uterine malformation and hysteroscopic treatment results. Hum Reprod Update 7(2):161-174

11. Grimbizis GF, Di Spiezio SA, Saravelos SH et al (2016) The Thessaloniki ESHRE/ESGE consensus on diagnosis of female genital anomalies. Hum Reprod 13(1):1-16

12. Grimbizis GF, Gordts S, Di Spiezio SA et al (2013) The ESHRE/ESGE consensus on the classification of female genital tract congenital anomalies. Hum Reprod 28(8):2032-2044

13. Homer HA, Li TC, Cooke ID (2000) The septate uterus: a review of management and reproductive outcome. Fertil Steril 73(1):1-14

14. Jurkovic D, Geipel A, Gruboeck K (1995) Three-dimensional ultrasound for the assessment of uterine anatomy and detection of congenital anomalies: a comparison with hysterosalpingography and two-dimensional sonography. Ultrasound Obstet Gynecol 5(4):233-237

15. Kasius JC, Broekmans FJM, Veersema S et al (2011) Observer agreement in the evaluation of the uterine cavity by hysteroscopy prior to in vitro fertilization. Hum Reprod 26(4):801-807

16. Knez J, Saridogan E, Van Den Bosch T et al (2018) ESHRE/ESGE female genital tract anomalies classification system-the potential impact of discarding arcuate uterus on clinical practice. Hum Reprod 33(4):600-606

17. Lagana AS, Ciancimino L, Mancuso A et al (2014) 3D sonohysterography vs hysteroscopy: a cross-sectional study for the evaluation of endouterine diseases. Arch Gynecol Obstet 290:1173-1178

18. Ludwin A, Ludwin I, Banas T et al (2011) Diagnostic accuracy of sonohysterography, hysterosalpingography and diagnostic hysteroscopy in diagnosis of arcuate, septate and bicornuate uterus. J Obstet Gynaecol Res 37(3):178-186

19. Ludwin A, Ludwin I, Kudla M et al (2014) Diagnostic accuracy of threedimensional sonohysterography compared with office hysteroscopy and its interrater/intrarater agreement in uterine cavity assessment after hysteroscopic metroplasty. Fertil Steril 101(5):1392-1399
20. Ludwin A, Ludwin I, Kudla M et al (2015) Reliability of the European Society of Human Reproduction and Embryology/European Society for Gynaecological Endoscopy and American Society for Reproductive Medicine classification systems for congenital uterine anomalies detected using three-dimensional ultrasonography. Fertil Steril 104(3):688-697

21. Ludwin A, Ludwin J (2015) Comparison of the ESHRE-ESGE and ASRM classifications of Mullerian duct anomalies in everyday practice. Hum Reprod 30(3):569-580

22. Ludwin A, Martins WP, Nastri CO et al (2018) Congenital Uterine Malformation by Experts (CUME): better criteria for distinguishing between normal/arcuate and septate uterus. Ultrasound Obstet Gynecol 51(1):101-109

23. Mitwally MFM, Abuzeid M (2008). Operative Hysteroscopy for Uterine Septum. In: Infertility and Assisted Reproduction. Editors: Botros R. M. Rizk, Juan A. Garcia-Velasco, Hassan N. Sallam and Antonis Nakrigiannakis. Cambridge University Press, Part II Infertility Evaluation and Treatment, Chapter 13,115-131.

24. Moini A, Mohammadi S, Hosseini R et al (2013) Accuracy of 3-Dimensional Sonography for Diagnosis and Classification of Congenital Uterine Anomalies. J Ultrasound Med 32:923-927

25. Raga F, Bauset C, Remohi J et al (1997) Reproductive impact of congenital Müllerian anomalies. Hum Reprod 12:2277-2281

26. Saravelos SH, Cocksedge KA, Li TC (2008) Prevalence and diagnosis of congenital uterine anomalies in women with reproductive failure: a critical appraisal. Hum Reprod Update 14(5):415-429

27. Smit JG, Kasius JC, Eijkemans MJC et al (2013) The international agreement study on the diagnosis of the septate uterus at office hysteroscopy in infertile patients. Fertil Steril 99(7):2108-2113

28. Smit JG, Overdijkink S, Mol BW et al (2015) The impact of diagnostic criteria on the reproducibility of the hysteroscopic diagnosis of the septate uterus: a randomized controlled trial. Hum Reprod 30(6):1323-1330

29. The American Fertility Society classifications of adnexal adhesions, distal tubal occlusion, tubal occlusion secondary to tubal ligation, tubal pregnancies, Müllerian anomalies and intrauterine adhesions (1988). Fertil Steril 49:944-55.

30. The American Society for Reproductive Medicine, Practice Committee of the American Society for Reproductive Medicine (2016) Uterine septum: a guideline. Fertil Steril 106(3):530-540

31. Yu LL, Zhang X, Zhang T et al (2014) Detection of congenital uterine malformation by using transvaginal three-dimensional ultrasound. J Huazhong Univ Sci Technolog Med Sci 34(5):782-784. https://doi.org/10. 1007/s11596-014-1352-7

\section{Publisher's Note}

Springer Nature remains neutral with regard to jurisdictional claims in published maps and institutional affiliations.

\section{Submit your manuscript to a SpringerOpen ${ }^{\circ}$ journal and benefit from:}

- Convenient online submission

- Rigorous peer review

- Open access: articles freely available online

- High visibility within the field

- Retaining the copyright to your article

Submit your next manuscript at $>$ springeropen.com 